Service social

\title{
La séparation et réunification familiales de dix femmes réfugiées congolaises
}

\section{Stéphanie Arsenault}

Volume 50, numéro 1, 2003

URI : https://id.erudit.org/iderudit/006924ar

DOI : https://doi.org/10.7202/006924ar

Aller au sommaire du numéro

Éditeur(s)

École de service social de l'Université Laval

ISSN

1708-1734 (numérique)

Découvrir la revue

Citer cet article

Arsenault, S. (2003). La séparation et réunification familiales de dix femmes réfugiées congolaises. Service social, 50(1), 122-144.

https://doi.org/10.7202/006924ar
Résumé de l'article

Cet article met en relief l'évolution des rôles de mère, de conjointe, de pourvoyeuse et de personne engagée dans la communauté de dix femmes congolaises réfugiées au Québec. Ces femmes ont toutes vécu un processus de séparation familiale prolongée et de réunification de leur famille en exil. Les principaux éléments de continuité sont les valeurs transmises et les stratégies adoptées pour l'éducation des enfants, la reprise d'une relation conjugale déjà satisfaisante au Congo, ou la rupture des couples boiteux avant l'exil, la responsabilité économique de la femme envers sa famille ainsi que la poursuite de l'engagement en exil pour les femmes déjà engagées chez elles. Les principaux éléments de rupture sont : la chute marquée du statut socioéconomique de la famille et la perte de l'exercice de la profession, l'atrophie de leurs réseaux sociaux et la détérioration des relations avec la famille élargie restée au Congo. 


\title{
La séparation et réunification familiales de dix femmes réfugiées congolaises
}

\author{
Stéphanie ARSENAULT \\ Étudiante au doctorat en anthropologie et travail social \\ Université de Granada (UGR) en Espagne \\ phanie36@hotmail.com
}

Cet article met en relief l'évolution des rôles de mère, de conjointe, de pourvoyeuse et de personne engagée dans la communauté de dix femmes congolaises réfugiées au Québec. Ces femmes ont toutes vécu un processus de séparation familiale prolongée et de réunification de leur famille en exil. Les principaux éléments de continuité sont les valeurs transmises et les stratégies adoptées pour l'éducation des enfants, la reprise d'une relation conjugale déjà satisfaisante au Congo, ou la rupture des couples boiteux avant l'exil, la responsabilité économique de la femme envers sa famille ainsi que la poursuite de l'engagement en exil pour les femmes déjà engagées chez elles. Les principaux éléments de rupture sont : la chute marquée du statut socioéconomique de la famille et la perte de l'exercice de la profession, l'atrophie de leurs réseaux sociaux et la détérioration des relations avec la famille élargie restée au Congo.

Mots clés : réfugiées, rôles, séparation familiale, réunification familiale

This article highlights the evolution of roles of mother, spouse, provider and person involved in the community in ten Congolese refugee women in Quebec. These women have each experienced a process of prolonged familial separation and subsequent reunification in exile. The principal elements of continuity are: the transmission of values and education strategies for their children, the continuance of a healthy conjugal relationship or the ending of an unhealthy conjugal relationship from the Congo, the economic responsibility of the woman towards her family, and the continuance of community roles in exile. Major elements of change: the pronounced socio-economic fall of the family, the inability to exercise their professions, the atrophy of their social networks, and the deterioration of relations with their extended families in the Congo.

Key words: refugees, roles, familial separation, familial reunification 


\section{INTRODUCTION}

Entre 1996 et 2000, le Canada a reçu en moyenne 206474 nouveaux arrivants par an. Environ 20000 d'entre eux ont été chaque année reconnus réfugiés au sens de la Convention de Genève. Le Québec accueille un peu plus du tiers de l'ensemble des réfugiés qui se réinstallent au Canada tous les ans. Son objectif annuel se chiffre à 7 600. Le nombre de l'ensemble des nouveaux arrivants accueillis par la province devrait quant à lui passer de 29772 reçus en 1996 (MRCl, 2001) à plus de 40000 en 2003 (MRCl, 2000).

Les ressortissants de la République démocratique du Congo (RDC), dans une très large majorité des réfugiés, constituaient environ $2,5 \%$ des nouveaux arrivants réinstallés au Québec en 2000. Au cours de la seconde moitié des années 1990, la RDC représentait le douzième principal pays d'origine des immigrants reçus par la province. Ainsi, près de 3000 Congolais (1 373 femmes et 1438 hommes) ont été admis par le Québec entre 1996 et $2000(\mathrm{MRCl}, 2001)$. Nombre d'entre eux ont été sélectionnés à l'étranger, tantôt en RDC, tantôt dans des camps de réfugiés situés en Tanzanie ou au Kenya notamment. D'autres ont plutôt été reconnus réfugiés au Canada par la Section de la protection des réfugiés de la Commission sur l'immigration et le statut de réfugié (CISR). Alors que les individus choisis depuis l'étranger pour s'installer au Canada sont généralement sélectionnés en famille, ceux qui quittent leur pays sans statut afin de revendiquer l'asile aux frontières canadiennes laissent souvent derrière eux les membres de leur famille immédiate en attendant le dénouement de leur demande de refuge et la possibilité de procéder à la réunification familiale. De nombreuses familles se trouvent ainsi temporairement fractionnées par les écueils de l'exil. Cette situation touche un nombre important de ressortissants congolais.

Dans le cadre de mon projet de recherche à la maîtrise en service social, j'ai choisi de m'attarder aux effets, perçus par quelques femmes réfugiées congolaises, de ces séparations familiales prolongées sur certains de leurs rôles au cours de la séparation et après la réunification en exil. J'expose ici l'essentiel des observations dégagées de cette étude. À cette fin, j'esquisse d'abord un bref portrait de la situation congolaise qui a poussé sur les routes de l'exil des centaines de milliers de Congolais. Je présente 
ensuite la démarche de recherche réalisée ainsi que le groupe de femmes interrogées. Enfin, je décris les principales observations retenues de cet exercice d'analyse.

\section{LES RACINES de LA VIOLENCE EN RÉPUBLIQUe DÉmOCRATIQUe DU CONGO}

Du point de vue des richesses naturelles que l'on retrouve sur son territoire (or, diamant, cobalt, cuivre, étain, coltan [colombotantalite], niobium, uranium, manganèse, bois précieux, café, etc.), la RDC est l'un des pays les plus riches d'Afrique, sinon de la planète. Or, depuis de nombreuses décennies, la violence ronge le tissu social du pays et sa population est devenue l'une des plus pauvres de notre époque. Cette situation a ses origines loin dans le passé, plus particulièrement dans l'aventure coloniale dont le pays fut l'objet. Mais un fil conducteur traverse depuis ce temps les chapitres de l'histoire congolaise : les richesses dont le pays regorge profitent à des acteurs nationaux ou étrangers qui laissent la population locale dans le plus grand dénuement.

Afin d'en retirer des dividendes et de contribuer par ce biais au développement de la Belgique, Léopold II, roi des Belges, s'intéressa, dès la fin du $19^{\mathrm{e}}$ siècle, aux abondantes ressources du Congo. Il devint propriétaire du pays en 1885 avant de remettre entre les mains de l'État belge les clés du Congo indépendant en 1908. Par la construction et la promotion de mythes sur la supériorité intellectuelle et génétique de certaines tribus, l'entreprise coloniale et civilisatrice qui s'ensuivit posa en terre africaine les ferments de la division socio-ethnique qui allait, quelques décennies plus tard, prendre tous les atours d'une guerre civile (Braeckman, 1996).

L'indépendance fut déclarée en 1960. L'enthousiasme avait gagné une large partie de la population aux premières heures de cette indépendance. C'était cependant sans savoir que le Congo allait prendre un chemin bien différent de celui dont rêvait le leader Lumumba avant de mourir assassiné par les forces de Mobutu six mois après sa nomination comme premier ministre (le premier) du pays. Une fois que Mobutu eut les reines du pouvoir bien en main, à la suite d'un violent coup d'État réalisé en 1965, un règne dictatorial caractérisé par une corruption et par une répression de tous les instants s'instaura. Dès l'aube des années 1990, alors que la population et les infrastructures du pays se trouvaient dans un 
état lamentable, la faillite du pouvoir en place commença à se faire sentir. La violence, sous forme de pillages, de massacres, de viols massifs, s'accentua de façon marquée dans tout le pays. Elle fut parfois orchestrée par le pouvoir central, et parfois le résultat de la dégradation de la situation économique et sociale du pays. Dans un tel contexte de décomposition générale, l'abondance des richesses décrites plus tôt ne manqua pas d'attirer sur le territoire une kyrielle d'acteurs prêts à tout pour accaparer la part du gâteau qui, selon eux, leur revenait. La première guerre du Congo menant à la fuite et à la mort de Mobutu éclata en 1997. Au moins six pays se trouvaient dès lors engagés dans le conflit. L'Angola, le Burundi, la Namibie, l'Ouganda, le Rwanda et le Zimbabwe s'engagèrent aux côtés des troupes de Laurent-Désiré Kabila dans l'entreprise visant à évincer Mobutu du pouvoir.

Une fois au pouvoir, Kabila prit soin d'éloigner de son entourage ceux qu'il jugeait désormais trop encombrants. Les forces rwandaises, burundaises et ougandaises se virent ainsi remerciées et congédiées. Devant cette rebuffade, les troupes "limogées " se joignirent aux groupes armés opposés au nouveau pouvoir en place. Ainsi, en août 1998 éclata la deuxième guerre du Congo. Une guerre qui mit en scène, d'une part, des milices tribales sous le giron de Kabila, les forces armées de ce dernier et les armées étrangères sympathisantes et, d'autre part, diverses factions opposées au pouvoir en place - également opposé entre elles auxquelles s'associèrent les forces étrangères désavouées par Kabila. De cette façon, depuis plusieurs années déjà, le pays est devenu, selon Tshiyembé, « ... la caricature d'une souveraineté confisquée par les puissances étrangères » (2000, p. 25). Quarante pour cent du territoire serait aujourd'hui sous contrôle étranger. Les uns contrôlent l'essentiel du commerce relié à l'exploitation du diamant, de l'or, des bois précieux ou du café dans les zones qu'ils assiègent. Les autres sèment la division entre certaines ethnies congolaises et fomentent les affrontements entre les groupes devenus opposés les uns aux autres. D'autres encore orchestrent, ou auraient orchestré, depuis le Congo, des manœuvres militaires visant à renverser le pouvoir en place dans leur pays d'origine (Burundi). Bref, le pays demeure marqué par la violence depuis le changement de gouvernement opéré en 1997. Des milliers de Congolais ont perdu la vie au cours de ces 
événements violents. Des centaines de milliers d'autres ont fui en exil dans les pays limitrophes ou outre-mer.

\section{DÉMARCHE ET QUESTION DE RECHERCHE}

C'est finalement sur cette toile de fond que sont accueillis au Québec des centaines de réfugiés congolais chaque année. Plusieurs d'entre eux demandent ici leur statut et doivent, pour ce faire, laisser derrière eux des membres de leur famille pour une période allant généralement jusqu'à trois ans. Pour mon étude, je me suis intéressée de façon particulière au vécu des femmes réfugiées dans un tel contexte de séparation familiale prolongée et de réunification en exil. J'ai donc voulu trouver des éléments de réponse à la question de l'évolution des rôles de certaines femmes réfugiées congolaises au cours du processus de séparation et de réunification familiales en exil. Ce sont plus spécifiquement leurs rôles de mère, de conjointe, de pourvoyeuse et de personne engagée dans la communauté qui ont retenu mon attention. Pour y arriver, j'ai rencontré dix femmes ayant vécu séparées d'un ou de plusieurs membres de leur famille immédiate pendant plus d'un an et qui sont maintenant réunies avec eux, ou certains d'entre eux, depuis au moins un an. Je me suis entretenue avec chacune pendant une heure trente en moyenne. Ces rencontres ont eu lieu à Québec, à Montréal et à Ottawa entre les mois de mai et de septembre 2001. J'ai pris contact avec les premières participantes par l'entremise d'organismes non gouvernementaux et par des réseaux d'Église. Ces femmes m'ont ensuite mise en contact avec d'autres Congolaises de leur connaissance qui vivaient la situation étudiée. Sept entretiens se sont réalisés au domicile même de la personne, un s'est tenu dans un restaurant, un dans un centre communautaire fréquenté par la dame et un autre a eu lieu au domicile d'un proche parent de la participante. Tous ces entretiens ont été enregistrés sur bandes audio, retranscrits, puis analysés à l'aide du logiciel Nudist-Vivo.

\section{LES FEMMES RENCONTRÉES}

Les femmes interviewées avaient en moyenne 46 ans au moment de l'entrevue, la plus jeune ayant 38 ans, la plus âgée, 54 ans. Au moment de quitter leur pays elles étaient toutes mariées. L'une d'elles est maintenant divorcée, une autre séparée. Ces femmes ont entre 4 et 10 enfants, pour une moyenne de 6,6 enfants par famille. Dans quatre des familles, certains de ces enfants ont été 
adoptés. II s'agissait d'enfants membres de la famille élargie qui se trouvaient en situation difficile ou encore d'enfants errants accueillis au sein de la famille de façon permanente. La moitié des femmes interrogées sont venues au Québec avant leur mari. Quatre sont au contraire venues rejoindre leur conjoint et les membres d'un couple ont fait le voyage ensemble, laissant pour un temps leurs enfants derrière eux. Trois des femmes rencontrées ayant précédé leur conjoint au Québec demeuraient, au moment de l'entrevue, séparées de celui-ci. Dans l'un des cas, il s'agissait d'une situation causée par certaines lenteurs administratives. Dans un deuxième cas, il s'agissait plutôt d'une entente mutuelle de continuer à vivre séparés, faute d'ouvertures professionnelles pour le mari médecin resté au Congo. Finalement, la troisième séparation était attribuable à un divorce envisagé dès le départ du pays. La durée moyenne de la séparation familiale s'établissait, pour cet échantillon, à trois ans.

\section{QUELQUES OBSERVATIONS IMPORTANTES}

Les rôles de la femme réfugiée d'origine congolaise se trouvent-ils modifiés dans un contexte de séparation familiale prolongée et de réunification en exil? Voilà la question ayant guidé la présente démarche de recherche. À la lumière de l'analyse effectuée, plusieurs éléments de réponse sont maintenant disponibles. Je présente ici les principales observations réalisées.

À la lecture des témoignages livrés par les répondantes, il apparaît rapidement combien ces femmes étaient, pour la plupart, des personnes actives et dynamiques au Congo. Plusieurs d'entre elles consacraient temps et énergie à des activités professionnelles, sociales et religieuses importantes en plus d'assumer leurs responsabilités familiales. Ces femmes étaient en majorité engagées dans une relation conjugale dont elles retiraient suffisamment de satisfactions pour souhaiter la voir perdurer. Elles étaient également satisfaites de l'exercice de leur rôle de mère de famille tel qu'il se présentait au Congo. Bref, avant que la violence ne vienne marquer leur existence, toutes les participantes s'affirmaient plutôt fières et contentes de la vie qu'elles menaient alors en Afrique. 


\section{DES PROCÉDURES ADMINISTRATIVES COMPLEXES ET LENTES}

Tout comme les études réalisées au Québec par Rousseau et ses collègues (1997 et 2001) et par Bertot et Mekki-Berrada (1999) l'avaient déjà révélé, la grande précarité dans laquelle arrivent au Canada les requérants du statut de réfugié rend ces derniers particulièrement susceptibles de souffrir de troubles mentaux comme l'anxiété et la dépression. Les femmes rencontrées ou leur mari ont tous demandé refuge une fois arrivés sur le territoire canadien et se sont par conséquent retrouvés, pour des périodes variables mais toujours supérieures à six mois, sans statut défini. Cette insécurité associée à leur condition statutaire et à l'absence de contrôle sur les délais subis pour ces démarches, comme pour les démarches visant la réunification familiale, a effectivement contribué à plonger plusieurs femmes dans un état de détresse psychologique flagrant.

Les femmes arrivées au Canada avant leur conjoint ou leurs enfants, ou avant une partie de ceux-ci, ont clairement évoqué la souffrance ressentie devant l'impossibilité de donner rapidement suite au projet de réunification familiale, le plus souvent en raison des délais administratifs et des coûts élevés exigés pour réaliser ce projet. Comme dans le cas de l'étude de Rousseau et de ses collègues (1997), la durée moyenne de la séparation familiale s'établissait à trois ans, mais elle a parfois atteint quatre ans. Dans les moments de désarroi devant le prolongement inattendu des délais menant aux retrouvailles, une femme dont les enfants l'ont rejointe après quatre ans d'attente affirmait que, si elle avait été au courant de la durée de ces délais, elle aurait préféré rester dans le danger avec ses enfants.

À la lumière de l'étude réalisée, il est possible d'affirmer, tout comme Renaud et Gingras (1998), que la lourdeur et la complexité des procédures administratives pour l'obtention du statut comme pour la réunification familiale portent atteinte à l'intégration harmonieuse des personnes touchées. Les efforts demandés pour accomplir la réunification sont tels que madame E., au Canada depuis trois ans avec maintenant cinq de ses huit enfants, considère toujours que les démarches pour compléter sa réunification familiale (la venue de son conjoint et de leurs trois autres enfants) constituent encore aujourd'hui son occupation principale. Les écueils rencontrés dans ce processus n'aident visiblement en rien 
l'expérience de séparation et l'exercice du rôle de mère rendu déjà laborieux par les difficultés de maintenir les contacts avec les enfants au loin et la situation chaotique dans laquelle ils se trouvent souvent pendant cette période d'attente. Cette même madame E. manifestait cependant, malgré toutes les difficultés éprouvées et les souffrances ressenties, une certaine fierté face à son sens de l'initiative et à son audace qui lui ont permis d'accomplir avec succès les premières démarches de réunification qui ont rendu possible la venue de deux de ses filles.

Les procédures administratives canadiennes et les coûts exigés sont aussi partiellement responsables des difficultés que pose le projet de faire venir les enfants adoptés qui faisaient partie de certaines familles congolaises avant l'exil. D'autres facteurs entrent aussi en jeu. Par exemple, les circonstances et les conditions dans lesquelles s'est effectuée la fuite du pays. La quasiimpossibilité d'amener en exil les enfants adoptifs demeure une lourde entrave au bien-être émotif des femmes touchées, même des années après la venue des enfants biologiques. Plusieurs femmes ont en ce sens soulevé la nécessité, à leurs yeux, de séparer le processus de réunification familiale pour les immigrants indépendants de celui pour les réfugiés reconnus au Canada, en raison notamment des conditions adverses dans lesquelles se trouvent généralement les membres encore au pays. II faudrait aussi, dans cette même perspective, faciliter les retrouvailles avec des enfants adoptés au Congo, même lorsque aucun papier officiel ne fait foi de cette adoption, comme c'est la norme en RDC.

Les défis de tous ordres posés aux femmes revendicatrices au Canada sont patents. Les femmes restées au Congo alors que leur mari venait faire les premières démarches pour demander refuge au Canada s'en sont visiblement mieux tirées que les autres pendant cette même période. Le soutien constant de la famille et de l'entourage, notamment sur le plan financier, semble être l'explication la plus probante. La parenthèse que représente la période de séparation, bien qu'elle soit douloureuse pour toutes, se vit donc différemment pour les mères restées au pays et pour celles venues en défricheuses chercher refuge pour leur famille au Canada.

Rousseau et al. (2001) affirmaient aussi que la souffrance ressentie pendant la séparation familiale, séparation sur laquelle les 
femmes ont bien peu de contrôle, mobilise leur univers de sens. La perception de la réalité qu'elles vivent pendant cette période se construit donc à travers le prisme des souffrances, des peurs et des pertes multiples avec lesquelles elles doivent composer depuis les troubles vécus au Congo et leur départ en exil. À cet égard, plusieurs femmes ont exprimé leur incapacité, durant cette période, de penser à d'autres projets personnels que celui de réunir leur famille. L'immense douleur qui découle de l'absence se trouve compensée par leur grand espoir et leur besoin de retrouver leurs enfants; on observe là les fondements de leurs efforts et de leurs sacrifices.

\section{DES CONDITIONS ÉCONOMIQUES DIFFICILES}

L'obligation de trouver rapidement une source de rémunération en exil amène généralement les femmes à accepter un emploi qui comporte des conditions de travail loin en deçà de ce qu'elles connaissaient auparavant au Congo. Alors qu'elles étaient médecin, infirmière, entrepreneure, professeure ou vendeuse au Congo, elles sont maintenant ouvrières en usine ou en manufacture pour la plupart. II y a donc lieu de se demander dans quelle mesure ces difficultés en ce qui concerne l'emploi marquent le parcours d'insertion globale à leur nouvelle société. Cette situation remet de plus en lumière l'enjeu crucial de la déqualification professionnelle des réfugiés qui empêche non seulement les réfugiés qualifiés d'exercer leurs compétences, mais également la société d'accueil de s'enrichir des expertises venues d'ailleurs.

Donc, sur le plan économique, plutôt que de découvrir ici la richesse et la consommation matérielle telles que notre société les valorise, c'est davantage la découverte de la pauvreté et de la survie au jour le jour qui devient le lot des réfugiées rencontrées. Mis à part les événements liés aux pillages et à la guerre qui ont précédé leur départ, c'est plutôt, pour ces femmes et leur famille, l'une des premières expériences de manque matériel et économique. La chute abrupte de statut socioéconomique qui s'ensuit représente un bien dur coup pour la plupart d'entre elles. II n'en demeure pas moins que, pour quelques-unes, cette même chute de statut devient l'occasion de mettre certaines valeurs en perspective et d'accorder désormais davantage d'importance aux relations familiales plutôt qu'à la poursuite du succès financier tel qu'elles l'ont connu en RDC. Plusieurs femmes ont exprimé leur grand 
sentiment d'impuissance et de dépression pendant cette période. II semble cependant que parfois, malgré les piètres conditions dans lesquelles elles travaillent, le fait de pouvoir exercer une activité quelconque à l'extérieur de leur appartement devient un moyen important pour parvenir à contrer en partie cet état de dépression et d'angoisse.

Si elles occupaient déjà une place active dans la subsistance de la famille au Congo, la plupart des femmes y ont pris une part cruciale dès la séparation familiale et elles la gardent après la réunification en exil. Alors que la majorité comptaient au Congo sur des revenus élevés et réguliers assurés par leur mari, par ellemême et par la famille élargie ou l'entourage au besoin, elles se retrouvent toutes avec des conditions économiques précaires, puisqu'elles doivent, de surcroît, relever le défi de repartir de zéro sur le plan matériel.

À la suite de la présente recherche, il apparaît aussi juste d'affirmer, comme de nombreux autres chercheurs l'ont exprimé dans le passé (Bertot et Bekki-Berrada, 1999; Blair, 2000; Hirayama et Cetingok, 1995; Lavik, Hauff, Skrondal et Solberg, 1996; Silove et al., 1997; Valtomen, 1999), que la précarité économique et l'absence d'emploi représentent des causes majeures de fragilité psychologique qui font obstruction à l'intégration sociale des réfugiés. Nombreuses ont été les femmes à affirmer à quel point les problèmes financiers ont perturbé et continuent de gêner leur dynamique familiale. Les nombreuses heures de travail supplémentaires, les horaires de nuit et les équipes toujours variables entravent le vécu familial, la femme ne pouvant passer, dans ces circonstances, que peu de temps avec ses enfants. Cette situation cause d'autant plus de problèmes qu'aucun autre membre de la famille ne peut les appuyer dans leurs responsabilités parentales, comme c'était généralement le cas au Congo. L'absence de travail, pour sa part, fait problème au moment de vouloir compter sur un logement suffisamment spacieux pour la famille nombreuse ou encore pour faire venir d'autres membres de la famille toujours en attente au pays.

Ces problèmes non seulement touchent le noyau familial en exil, mais ils se répercutent aussi sur la dynamique avec la parenté encore au pays. L'impossibilité d'appuyer financièrement la famille élargie restée au pays constitue pour beaucoup une déception 
majeure et un facteur qui porte atteinte à la qualité des liens entretenus avec les autres membres de la famille. Une étude de Bertot et Jacob (1991) avait déjà affirmé qu'effectivement les pressions exercées par la famille restée à l'étranger sur ses membres en exil dans le but de recevoir un appui financier pèsent lourd, que les rapports au sein de l'ensemble familial changent et que, le plus souvent, ils se détériorent.

\section{UN ACCÈS DIFFICILE AUX CARRIÈRES PROFESSIONNELLES}

À l'instar des études de Valtomen (1999) et de Rousseau et al. (2001), la présente recherche montre que, pour la période étudiée, l'état de la conjoncture économique au Québec marquée par un haut taux de chômage, combiné avec les conditions d'accession aux emplois gérés par des ordres professionnels spécifiques, s'est avéré plus déterminant encore que le degré de scolarité prémigratoire dans le processus d'intégration des femmes et de leur mari sur le marché du travail. En fait, toutes les femmes rencontrées, qu'elles aient fait ou non des études universitaires ou techniques, se sont retrouvées ici dans des emplois du secteur ouvrier ou domestique, la plupart jusqu'au moment de l'entrevue.

L'enjeu de la déqualification professionnelle se présente donc aussi de façon criante dans les résultats de ma recherche. Plusieurs des femmes interviewées possèdent une formation universitaire ou professionnelle acquise au Congo, mais aussi parfois en Europe. Malgré ce fait, elles se sont toutes heurtées à une fin de non-recevoir au moment de vouloir intégrer le marché du travail dans leur domaine respectif au Québec. La complexité des démarches et les coûts exigés pour faire accréditer des compétences ou des diplômes étrangers semblent agir de façon dissuasive sur les personnes en quête d'une telle reconnaissance, puisque aucune des répondantes n'avait encore choisi de lutter jusqu'au bout afin d'obtenir cette équivalence. Plusieurs avaient cependant décidé, avec un enthousiasme certain, d'entreprendre des études connexes à leur domaine afin de retrouver un jour un statut professionnel satisfaisant.

Ce constat au sujet de la non-reconnaissance des compétences professionnelles acquises à l'extérieur du Canada, particulièrement dans les pays du tiers-monde, semble aller dans le sens des conclusions de Nguyen (1982) sur ce même point. Celui-ci affirmait que la perte du statut professionnel en soi avait un impact 
sensible sur la personne touchée. Cet impact pourrait être aussi important, et même plus important, que la condition économique absolue, c'est-à-dire la valeur des revenus dont elles disposent. Deux des participantes ont formulé des commentaires particulièrement évocateurs à ce sujet. Madame A. affirmait entre autres choses que, pour des gens sans compétences professionnelles, la possibilité de recevoir ici une formation technique ou d'intégrer un emploi en usine représentait sûrement un gain par rapport à ce qui avait été vécu sur le même plan au Congo. Elle poursuivait en affirmant qu'au contraire, pour les personnes arrivées avec des compétences ou des formations spécifiques, l'impossibilité de mettre à profit ces acquis devient une source majeure de frustrations et de déceptions. Madame E. renchérissait sur ce point par des propos tout aussi révélateurs:

Et puis j'ai aussi remarqué que la dépression est plus forte pour une femme professionnelle, parce que non seulement tu as perdu ton mari, ta famille, plus ton travail. Oui, tandis que pour la plupart de mes consœurs, c'était juste ta famille, plus ton mari. Mais pour nous, c'était ton travail, ta raison de vivre, ta propre estime de soi, parce que tu avais lutté pour y arriver. Donc ça aussi c'est dur!

Malgré ce constat, plusieurs femmes touchées par cette réalité ont manifesté leur espoir et leur volonté de lutter pour acquérir ici un statut dont elles seraient satisfaites. Elles ont notamment abordé la question en mentionnant que cela représentait pour elles un certain recommencement et que, petit à petit, elles reconstruiraient une situation qui les rendrait fières des efforts déployés. Mesdames G. et I. mentionnaient par exemple qu'au Congo, déjà, elles avaient dû lutter pour bâtir ce qu'elles avaient acquis de richesses et que, de la même façon, elles devraient ici monter une marche à la fois afin d'atteindre une qualité de vie conforme à leur convenance. Madame $\mathrm{B}$. racontait quant à elle que ce fut seulement après une période de révolte contre l'impossibilité d'exercer son métier en exil qu'elle décida finalement avec enthousiasme d'entreprendre des études dans un nouveau domaine.

\section{L'IMPORTANCE DE L'ENTOURAGE}

Le soutien social et familial représente une autre dimension importante à considérer dans l'évaluation des ressources sur lesquelles comptent les personnes réfugiées pour développer leur 
intégration à leur nouvelle société (Barclay, 1998; Bertot et Jacob, 1991; Blair, 2000; Duval, 1992; Hirayama, Hirayama et Cetingok, 1993; Jacob, 1994; Rousseau, Moreau, Drapeau et Marotte, 1997). Toutes ces recherches ont affirmé le caractère déterminant de la présence d'un réseau social pour faciliter aux réfugiés la reconstruction de leur vie en exil. L'existence d'un réseau de personnes de même origine serait particulièrement nécessaire dans les premiers temps de la réinstallation en exil. II n'en va pas autrement pour les femmes congolaises rencontrées. La perte de la famille élargie, laquelle est généralement très présente dans le déroulement de la vie quotidienne au Congo, se fait sentir de façon marquée au Québec. Cette perte crée donc un vide considérable pour plusieurs répondantes, vide qu'elles essaient parfois de combler par des rapports avec des compatriotes rencontrés dans les lieux de culte qu'elles fréquentent ou encore dans leurs milieux de travail. Plusieurs tissent ainsi des liens très serrés avec des familles amies qui deviennent en quelque sorte les oncles, tantes et cousins substituts de leurs enfants. Mais, comme l'affirme l'une d'elles, les amis, même les plus proches, ne remplacent jamais complètement sa propre famille restée au pays.

Toujours au sujet du soutien social, madame A. exprimait clairement combien sa détresse était grande et à quel point elle était inconsolable jusqu'au jour où une religieuse l'aborda dans la rue alors qu'elle pleurait. Cette religieuse devint, dès lors, son principal soutien moral pour affronter les épreuves que représentaient la séparation, la recherche d'emploi et les démarches liées à la réunification familiale. Plusieurs autres femmes ont également montré l'importance qu'elles accordent encore aujourd'hui aux personnes et aux organismes qu'elles ont fréquentés ou qu'elles continuent de fréquenter. En contrepartie, la solitude et l'isolement sont généralement présentés comme des réalités difficiles à accepter et à assumer, et même comme des atteintes à leur bienêtre affectif et physique. Les femmes qui ne peuvent compter sur un voisinage ami en souffrent beaucoup. Le fait que des personnes de confiance partagent les joies et les peines de la vie de tous les jours représente un atout quasi indispensable.

Dans le cas des personnes rencontrées, ce sont souvent les milieux d'Église qui deviennent les éléments du réseau social les plus importants et les plus significatifs. Rares sont les femmes qui affirment ne pas fréquenter un groupe religieux de façon au moins 
hebdomadaire. Les enfants participent généralement à ce vécu communautaire et spirituel. J'apporterais cependant un bémol à cette donnée qui veut que l'importance de la religion soit déterminante pour les femmes congolaises. II se pourrait que cette réalité soit en fait due à un biais de mon échantillonnage, puisque la majorité des participantes m'ont été présentées par une personne fort engagée dans les milieux d'Église. Ainsi, les répondantes que j'ai interrogées viennent peut-être justement en bonne partie d'un réseau d'Église. Par contre, les participantes rencontrées par l'entremise d'autres personnes contacts manifestaient le même enthousiasme par rapport à la place que la religion occupe dans leur vie.

\section{VIVRE LOIN LORSQUE DES ÉVÉNEMENTS MAJEURS SURVIENNENT DANS SON PAYS}

D'autres éléments viennent perturber l'équilibre psychologique des femmes réinstallées en exil. Blair (2000) avait notamment relevé que la perte d'un proche parent dans des circonstances reliées à la guerre était l'un des principaux déclencheurs de troubles mentaux majeurs en exil. Deux des participantes ont effectivement exprimé des sentiments de grande souffrance en évoquant la mort de la mère, dans un cas, et celle d'une sœur particulièrement appréciée, dans l'autre cas. Dans le discours des répondantes, ces événements survenus en période de guerre, en leur absence, sont directement associés à des problèmes émotifs majeurs expérimentés au Québec par la suite. La perte du sommeil, la perte de l'appétit, l'apparition de difficultés persistantes pour communiquer avec les enfants et d'un sentiment prolongé de dépression y sont pour elles directement associées. C'est donc dire que la dynamique familiale et l'exercice du rôle de mère peuvent se trouver lourdement affectés lorsque des événements traumatiques se produisent auprès des proches de la famille restés au Congo. Dans des situations comme celles-ci, la prière et le soutien de compatriotes ou d'amis représentent encore une fois un atout de taille.

\section{DES ÉLÉMENTS DE CONTINUITÉ}

Plusieurs éléments de continuité sont apparus dans les différents rôles de la femme. Le vécu conjugal est peut-être le rôle le plus clairement marqué par cette continuité entre un avant et un après qui se rejoignent sur des bases communes. J'abonderais donc ici 
dans le sens des propos de Jacob, Jouthe et Forcier (1999) qui affirmaient, à la suite de leur recherche, que les facteurs de rupture et de continuité dans le couple se retrouvent au sein même de la dynamique existante depuis le pays d'origine et qu'ils ne sont pas liés comme tels au processus migratoire.

Les principaux motifs de mécontentement évoqués par les deux femmes concernées par des relations peu satisfaisantes au Congo étaient principalement associés aux relations extraconjugales du mari. II apparaît donc que la continuité dans le couple repose sur des atouts présents avant la migration. Suivant la même logique, les problèmes présents avant l'exil deviennent précurseurs des problèmes à venir dans le couple après la migration ou de la rupture à survenir.

Ainsi, les retrouvailles conjugales se poursuivent généralement dans le sens de la dynamique qui existait auparavant. Par ailleurs, les expériences, même positives, demandent une période de réadaptation et de réapprivoisement mutuels. Malgré la hâte et le désir de se retrouver, les longues périodes de séparation provoquent souvent chez la femme un sentiment de peur et d'incertitude face aux retrouvailles à venir. Madame E., toujours séparée de son mari depuis maintenant trois ans, l'exprimait clairement en affirmant que son mari est maintenant devenu presque un étranger pour elle et ses enfants. Madame G. affirmait, quant à elle, qu'avant l'arrivée de son mari elle espérait ardemment son retour, tout en craignant quelque peu la perte des libertés nouvellement acquises pendant son absence. C'est en ce sens que la réunification semble parfois vécue comme un étrange mélange de joie et de crainte face à une certaine perte de ce qui s'était mis en place au cours de la séparation (Rousseau et al., 2001).

Rousseau et al. (2001) affirmaient que la dynamique familiale fonctionne selon cette même logique, c'est-à-dire que son harmonie et son devenir en exil reposent sur les atouts présents dès le pays d'origine. Elles affirmaient que le rétablissement de la famille dépend de la «... possibilité de continuer de s'appuyer sur les systèmes de références et de valeurs qui leur ont permis jusquelà de construire leur vie et de traverser les moments difficiles, c'est-à-dire la possibilité de s'appuyer sur ce que nous désignons par "tradition" » (2001, p.197). 
Sur ce point, les résultats de ma propre recherche ne sont pas aussi clairs, mais certains éléments permettent tout de même d'entrevoir une tendance en ce sens. Comme je l'ai mentionné maintes fois déjà, les femmes rencontrées affirment effectivement miser de façon importante sur des stratégies d'éducation et d'encadrement des enfants qui leur viennent de leur vie au Congo. Elles y accordent d'ailleurs beaucoup d'importance. Les rencontres et les prières en famille sont deux exemples de ces méthodes éducatives transposées au Québec après la réunification familiale. Cependant, une femme séparée de ses enfants pendant quatre ans dépeint une situation différente. Elle exprime clairement comment il lui a été impossible de reprendre les balises éducatives utilisées auparavant auprès de ses enfants. Une fois arrivés au Québec, ceux-ci n'ont désormais plus voulu accepter son autorité de mère telle que conçue en RDC. La durée particulièrement longue de la séparation (quatre ans) et les conditions de vie difficiles dans lesquelles ont vécu ces enfants pendant cette longue période ont pu influencer ce dénouement. Effectivement, si l'on en croit les résultats de l'étude de Duval (1992), plus la période de séparation s'avère longue, plus la reprise des contacts avec les enfants peut se révéler pénible. Les résultats de ma recherche semblent aller en ce sens.

L'évocation de cet exemple m'amène également à parler de la perte de confiance ressentie envers leurs parents en exil par des enfants restés au Congo et qui s'est produite au sein de quelques familles. II est opportun d'analyser combien cette perte de confiance s'est avérée dévastatrice dans certains cas, dont celui de madame J. qui a finalement perdu tout contrôle sur ses enfants après leur arrivée. Sa situation s'avère particulièrement difficile si l'on considère que c'est souvent précisément l'espoir dans l'avenir des enfants qui constitue le fer de lance des projets familiaux. Tous les efforts sont généralement faits par les parents pour garantir un avenir prometteur à leurs enfants (Jacob et al., 1994). Les nombreux efforts effectivement déployés par cette femme pendant quatre ans de labeur acharné, soutenus par un espoir intense de retrouver ses enfants, ont été amèrement déçus une fois les enfants arrivés au Québec. C'est sa foi indéfectible en Dieu qui lui permet maintenant d'entretenir l'espoir de réunir un jour ses enfants auprès d'elle. 
D'autres cas moins dramatiques illustrent eux aussi une perte de confiance certaine qui semble vouloir perdurer assez longtemps après les retrouvailles. Madame A. parlait notamment de la communication avec sa fille, qui n'a jamais repris l'allure qu'elle avait avant la séparation, même un an et demi après les retrouvailles. II faut cependant se demander, dans ce cas précis, si ce n'est pas le passage à l'adolescence de la jeune fille pendant cette période qui occasionne la transformation de la communication décrite entre la mère et la fille, transformation dans laquelle la mère ne reconnaît plus très bien son rôle.

Dans la même perspective, plusieurs femmes ont abordé avec émotion leur absence auprès de leurs enfants à des moments particuliers de leur existence. Deux femmes ont entre autres parlé de la puberté de leurs filles au moment où elles étaient loin d'elles, ou encore du passage à l'adolescence de leurs garçons, toujours en leur absence. II semble que le fait d'avoir raté ces moments importants marque de façon majeure ces mères de famille congolaises. Les femmes touchées en gardaient encore, au moment de l'entrevue, un souvenir vif et amer. Elles en gardent le sentiment de ne pas avoir assumé entièrement leurs responsabilités de mère.

Dans un autre registre, certains chercheurs affirment que l'origine rurale des réfugiés représente un facteur susceptible de compliquer l'intégration des réfugiés à leur nouvelle société, particulièrement pour ce qui touche la recherche d'un emploi (Valtomen, 1999; Nicholson, 1997). II faut donc voir que, dans la présente étude, toutes les femmes, sauf une, vivaient dans de grands centres urbains au moment de quitter le Congo. Certaines d'entre elles étaient originaires des régions rurales, mais elles habitaient depuis longtemps la ville. De surcroît, elles faisaient presque toutes partie d'une classe économique relativement privilégiée. Ces deux éléments, à n'en point douter, auront influencé leur façon de vivre, tant au Congo qu'au Québec, de même que leurs différents rôles mis ici en relief. II pourrait donc s'avérer intéressant d'approfondir ces mêmes questions auprès de femmes originaires de milieux ruraux et de conditions économiques plus modestes afin de voir si ces facteurs influencent effectivement l'intégration et les rôles des femmes congolaises en exil et, si tel est le cas, dans quelle mesure ils le font. 
Je reprends ici les propos de Duval qui concernaient plus spécifiquement les femmes au foyer mais qui sont, après mon étude, toujours pertinents pour les femmes réfugiées, qu'elles travaillent ou non à l'extérieur de leur foyer. Duval affirmait ainsi que « ... de façon générale, les conditions dans lesquelles ces femmes doivent exercer leur fonction de mère et d'épouse changent radicalement quand elles arrivent aux Québec » (1992, p. 11). De la même manière, leurs rôles de pourvoyeuses et de personnes engagées dans la communauté s'exercent au Québec dans des conditions toutes différentes de leur expérience congolaise. Quoi qu'il en soit, il ressort nettement que les forces et les outils dont faisaient usage avant l'exil les femmes rencontrées leur servent d'assises pour cheminer de façon constructive dans leur processus d'intégration à leur nouvelle société.

Je termine donc maintenant avec un clin d'œil du côté de l'intervention auprès de la population réfugiée. Summerfield (1999) demeure peut-être l'un des opposants les plus convaincus aux approches " victimisantes » et " pathologisantes " souvent utilisées dans l'intervention auprès des personnes touchées par la persécution, la guerre et l'exil. II propose plutôt, comme Bertot et Jacob (1991) et de Duval (1992) notamment, une perspective d'intervention mettant l'accent sur le pouvoir collectif de reconstruction de ces personnes (Summerfield, 1999, p. 1449). II invite aussi à s'écarter d'un glissement souvent observé en Occident : celui qui fait des situations sociales problématiques des problèmes d'ordre biopsychomédical et qui enferme de surcroît trop souvent les personnes touchées par l'exil et ses aléas dans un rôle de victimes passives, consommatrices de services fournis par des experts. En accord avec cette mise en garde, je propose aussi une perspective d'intervention dans laquelle le dynamisme, les forces et les capacités collectives des femmes réfugiées devraient servir d'assises à toute démarche d'analyse et de résolution des situations problématiques rencontrées par celles-ci dans leur processus d'intégration à leur nouvelle communauté. 


\section{CONCLUSION}

Les femmes réfugiées congolaises sont nombreuses à vivre une séparation familiale prolongée dans leur processus de migration et de recherche d'un refuge au Canada. Beaucoup de Congolais quittent leur pays en laissant temporairement derrière eux conjoint et enfants afin de trouver à l'étranger une terre où échapper à la guerre et où reprendre racine. Une recherche effectuée récemment au Québec auprès de cette population par la Table de concertation des organismes au service des personnes réfugiées et immigrantes au Québec (TCRI), en collaboration avec le Centre universitaire de santé de McGill et l'Équipe de recherche et d'action en santé mentale et culture (ÉRASME), a permis de mettre en lumière certains éléments de la réalité que ces familles vivent une fois établies au Québec. La présente étude visait à approfondir certaines autres dimensions de la vie de femmes congolaises en exil.

À la question de l'évolution des rôles de mère, de conjointe, de pourvoyeuse et de personne engagée dans la communauté des femmes réfugiées confrontées à une séparation familiale prolongée, les réponses sont multiples et nuancées. II ressort que la vie des femmes en exil, de même que l'exercice de leurs rôles, s'inscrit, pour un certain nombre de points, en continuité avec leurs expériences congolaises respectives. En quelques mots, disons que ces femmes utilisent les forces et les aptitudes qu'elles possèdent depuis le Congo afin de retrouver en exil un certain équilibre personnel, conjugal et familial. Néanmoins, il appert également que le contexte dans lequel s'actualisent les rôles des femmes rencontrées s'avère substantiellement modifié et qu'il influence alors de façon notoire la réalisation de ces rôles.

Les principaux éléments de continuité relevés dans les témoignages vont comme suit: le maintien des valeurs transmises notamment la déférence envers les aînés, le respect de l'autorité parentale et la pratique religieuse - et des stratégies adoptées pour l'éducation et l'encadrement des enfants, la poursuite d'une relation conjugale satisfaisante depuis le Congo et la rupture de couples déjà boiteux avant l'exil, l'engagement économique de la femme en ce qui concerne la subsistance de sa famille et la 
poursuite de l'implication au Québec pour les femmes déjà engagées socialement chez elles.

Les principaux éléments de rupture avec le passé se retrouvent, pour leur part, dans la chute marquée du statut socioéconomique des familles une fois au Québec, dans la perte de la reconnaissance professionnelle acquise antérieurement à l'exil, dans la perte d'un réseau social souvent actif comme soutien moral et financier dans des moments de besoin, dans la détérioration des relations avec la famille élargie restée au Congo, de même que dans l'interruption des soins portés à des enfants adoptifs autrefois intégrés au sein du noyau familial.

Plusieurs limites à l'étude peuvent être relevées. Je les regroupe ici afin de bien saisir la portée de mes résultats. D'abord, le petit nombre de femmes formant l'échantillon permet d'envisager de nouvelles hypothèses de recherche et de signaler des pistes d'explication à vérifier par des recherches ultérieures plus poussées. Cependant, il n'autorise pas la généralisation des résultats obtenus. Dans un autre ordre d'idées, certains thèmes qui auraient pu s'avérer importants dans l'exploration de la situation des femmes réfugiées congolaises n'ont pas été abordés, faute de temps et des conditions nécessaires pour le faire. Je pense ici surtout aux sévices sexuels dont les femmes auraient pu être victimes lors des pillages et des massacres ou à la violence conjugale qu'elles auraient également pu vivre au Congo et au Québec. Aucune question n'a été posée aux femmes sur ces sujets précis. Ces dimensions n'ont donc pu être prises en compte dans l'analyse des résultats.

Aussi, la présence du mari et du fils aîné au cours de deux entrevues a certainement teinté la couleur des propos échangés à cette occasion. J'ai cependant choisi de composer avec la présence de ces hommes malgré la difficulté de mesurer l'incidence sur les propos échangés - de la présence de tierces personnes imposée par la situation.

Je l'ai mentionné plus tôt, quatre femmes étaient, au moment de l'entrevue, toujours séparées de leur mari ou de certains de leurs enfants, encore à l'étranger. Le nombre d'entretiens réalisés avec une femme réunie avec l'ensemble des membres de sa famille est donc plus réduit que celui visé originellement. Un autre aspect important déjà signalé concerne les attributs socioéconomiques 
des participantes. Presque toutes ces femmes venaient de milieux urbains et appartenaient, au Congo, à une certaine élite économique. Il est donc important de tenir compte de toutes ces données qui auront eu une certaine incidence sur le vécu des femmes en exil ainsi que sur leur façon de l'exprimer.

Les résultats de cette recherche permettent de poser plusieurs nouvelles questions de grand intérêt. II serait à mon sens fort pertinent d'approfondir la question de la déqualification professionnelle des personnes réfugiées au Québec. II serait entre autres intéressant de compter sur un portrait qui permette de savoir combien de personnes sont affectées par cette situation et quelles sont les professions les plus difficiles à intégrer à l'heure actuelle. Sur la même question, mais dans une autre perspective, il m'apparaît tout aussi pertinent d'approfondir, avec les personnes touchées par cette problématique, les effets perçus de cette déqualification professionnelle sur les différentes sphères de leur vie. II pourrait ainsi se révéler plus facile de comprendre les incidences réelles de ce phénomène sur l'existence des principaux intéressés.

D'autres avenues de recherche émergent également de cette étude. Les effets, sur la vie des réfugiés, de la détérioration ou de la perte des relations avec la famille élargie restée dans le pays d'origine sont une avenue de grand intérêt. II serait aussi fort utile de donner la parole aux hommes afin d'approfondir leur propre vécu d'une séparation familiale en contexte de recherche de refuge. Voilà donc quelques pistes de recherche pertinentes à emprunter pour des projets futurs. 


\section{RÉFÉRENCES BIBLIOGRAPHIQUES}

BARCLAY, H.H. (1998). "Validating torture refugees: Reconnection in social work policy and practice », International Social Work, vol. $41, n^{\circ} 1$, p. $211-226$.

BERTOT, J. et A. JACOB (1991). Intervenir avec les immigrants et les réfugiés, Montréal, Méridien.

Bertot, J. et A. MeKki-BerRada (1999). Des services aux demandeurs d'asile : Pourquoi? Ce qu'en disent les intervenants d'organismes communautaires du grand Montréal, Table de concertation des organismes au service des personnes réfugiées et immigrantes (TCRI).

BLAIR, R.G. (2000). " Risk factors associated with PTSD and major depression among Cambodian refugees in Utah », Health and Social Work, vol. 25, $n^{\circ} 1$, p. 23-30.

BRAECKMAN, C. (1996). Terreur africaine. Burundi, Rwanda, Zaïre : les racines de la violence, Paris, Fayard.

DuvaL, M. (1992). Être mère au foyer à Montréal... quand on arrive de l'étranger, Québec, ministère des Communautés culturelles et de I'Immigration (Études et recherches $\mathrm{n}^{\circ} 1$ ).

HiRayama, H. et M. Cetingok (1995). "Amerasian refugees: Social characteristics, service needs, and mental health », Journal of Sociology and Social Welfare, vol. 22, n 4, p. 69-84.

HiRayama, K.K., H. Hirayama et M. CetingoK (1993). "Mental health promotion for Southeast Asian refugees in the USA », International Social Work, vol. 36, n 2, p. 119-129.

JACOB, A.G. (1994). "Social integration of Salvadoran refugees », Social Work, vol. 39, n 3 , p. 307-312.

JACOB, A., E. JOUTHE et L. FORCIER (1999). Facteurs de rupture et de continuité chez des couples immigrants et réfugiés, Montréal, Université du Québec à Montréal, FCAR.

LAVIK, N.J., E. Hauff, A. SkRondal et O. Solberg (1996). " Mental disorder among refugees and the impact of persecution and exile: Some findings from an out-patient population », British Journal of Psychiatry, 169, p. 726-732.

Ministère des ReLATIONS AVEC LES CITOYENS ET DE L'IMMIGRATION (2000). Planification de l'immigration permanente au Québec pour la période 2001-2003, Québec, Gouvernement du Québec. 
MINISTĖRE DES RELATIONS AVEC LES CITOYENS ET DE L'IMMIGRATION (2001). Tableaux sur limmigration au Québec, 1996-2000, Québec, Gouvernement du Québec.

NGUYEN, S.D. (1982). "The psycho-social adjustment and the mental health needs of Southeast Asian refugees ", The Psychiatric Journal of the University of Ottawa, vol. 7, $n^{\circ} 1$, p. 26-35.

NiCHOLSON, B.L. (1997). "The influence of pre-emigration and postemigration stressors on mental health: A study of Cambodian refugee », Social Work Research, vol. 21, n 1, p. 19-31.

RENAUD, J. et L. GINGRAS (1998). Les trois premières années au Québec des requérants du statut de réfugié régularisés, 2, Québec, Gouvernement du Québec.

Renaud, J., L. Gingras, S. Vachon, C. Blaser, J.-F. Godin et B. Gagné (2001). Ils sont maintenant d'ici ! Les dix premières années au Québec des immigrants admis en 1989, Québec, ministère des Relations avec les citoyens et de l'Immigration.

Rousseau, C., J. Bertot, A. Mekki-Berrada, T. Measham et A. DraPEAU (2001). Étude longitudinale du processus de réunification familiale chez les réfugiés (RS 2963 096), Montréal, Conseil québécois de la recherche sociale.

Rousseau, C., S. Moreau, A. Drapeau et C. Marotte (1997). Politique d'immigration et santé mentale. Impact des séparations familiales prolongées sur la santé mentale des réfugiés, Montréal, Conseil québécois de la recherche sociale.

Silove, D., S. Sinnerbrink, A. Field, V. Manicavasagar et Z. Steel (1997). " Anxiety, depression and PTSD in asylum-seekers, associations with pre-migration trauma and post-migration stressors », British Journal of Psychiatry, 170, p. 351-357.

SUMmeRFIELD, D. (1999). " A critique of seven assumptions behind psychological trauma programmes in war-affected areas », Social Science and Medicine, 48, p. 1449-1562.

TSHIYEMBÉ, M. (2000). "Ambitions rivales dans les Grands Lacs », Manière de voir, 51, p. 22-26.

VALTOMEN, K. (1999). "The societal participation of Vietnamese refugees: Case studies in Finland and Canada », Journal of Ethnic and Migration Studies, vol. 25, $n^{\circ} 3$, p. 469-491. 